\title{
SCORING LIGAND EFFICIENCY
}

\author{
JAROSLAW POLANSKI ${ }^{1 *}$, ROKSANA DUSZKIEWICZ ${ }^{1,2}$, ANNA PEDRYS $^{1}$ \\ and JOHANN GASTEIGER ${ }^{3}$
}

\author{
${ }^{1}$ Institute of Chemistry, University of Silesia in Katowice, Szkolna 9, 40-006 Katowice, Poland \\ ${ }^{2}$ Department of Pharmacology, School of Medicine in Katowice, \\ Medical University of Silesia in Katowice, Medyków 18, 40-752 Katowice, Poland \\ ${ }^{3}$ Computer-Chemie-Centrum, University of Erlangen-Nuernberg, Erlangen 91052, Germany
}

\begin{abstract}
Ligand efficiency (LE) is a molecular descriptor that probes the ratio of potency vs. heavy atom count (HAC). As an estimator of drug candidates, LE emphasizes a low heavy atom count more than potency. The objective was to design a novel transform where potency and the HAC would be balanced more evenly. A series of novel descriptors SCORE were defined to evaluate the co-influence of potency and the HAC. In particular, the product ligand efficiency (PLE) was designed and tested using the data of the ChEMBL, PubChem as well as the selected series of drugs and drug-fragments.
\end{abstract}

Keywords: ligand efficiency, product ligand efficiency: heavy atom count, activity, drugs, drug design, PubChem, ChEMBL

In drug design, we are optimizing the binding ability of ligands as a function of their chemical structure. A variety of methods has appeared that focus on this problem. In the simplest approach, the chemical structure can be represented by molecular size. The importance of molecular size in drug development has received much attention $(1,2)$. Generally, an increase in the molecular size also increases the molecular complexity. It is interesting to analyze how this increase affects the probability of identifying new drugs. In particular, the chances of finding smaller and less complex ligands is higher than it is for larger ones. In turn, an increase in molecular complexity can also increase the potency of ligand-target binding. Eventually, the binding can drop below a measurable level for molecules or fragments that are too small $(3,4)$. In other words, ligand size plays a dichotomic role in matching and binding a target. Rating these effects is an important tool in the search for a more efficient way to design better drugs. The heavy atom count (HAC) is a simple descriptor measuring molecular size that is related to ligand efficiency (LE), which is commonly used to evaluate the binding ability of ligands. LE is defined as the ratio of binding energy to the HAC (1, 5-8). A variety of LE-based analyses have been proposed (1) despite the fact that the observed trend of
LE seemed to be paradoxical and therefore could not be fully understood. (9-15). Not only has the mathematical validity of LE with its high preference for small ligands been questioned but a number of authors have indicated that this descriptor could have a quite low degree of usefulness $(9,10,13-15)$. e.g., being "uninformative when the changes do not significantly alter the size of the compounds" (16). In contrast, generally, LE has gained an enthusiastic reception (1). One of the precautions sounds however that LE is size-dependent and therefore we should not compare the compounds of extremely different sizes, which sounds accurately opposite to the warning (16). Accordingly, this is the reason for the uncertainties connected with LE.

Essentially, LE was designed to evaluate the average contribution of one non-hydrogen atom (HAC) to the binding free energy. We can easily understand the importance of the direct relation of LE to binding energy, if we recognize that LE is commonly calculated as a function of the inhibitory concentration $\mathrm{pIC}_{50}$, namely, $\mathrm{LE}=\mathrm{pIC}_{50} *(1.37 / \mathrm{HAC})$ (1). Accordingly, the closely related ligand efficiency index (LEI), which is defined directly by the $\mathrm{pIC}_{50} / \mathrm{HAC}(1)$, seems to be a simple replacement for LE; however, LEI is rarely discussed in the literature.

\footnotetext{
* Corresponding author: e-mail: jaroslaw.polanski@us.edu.pl
} 
To explain the paradoxical LE behavior, we should realize the dichotomic nature of the molecular vs. molar representations of chemical compounds (13, 14). Molecular descriptors (MD) and properties (P) are the best illustration here. While molecular descriptors are usually calculated from the representation of a single molecule, the properties are usually measured in experiments for substances, i.e. for ensembles of molecules. Sometimes, these representations are barely distinguishable (17). In this context, on the one hand, LE has been designed to be a molecular descriptor that is connected with a single molecule or, more precisely, with a single HAC that is a part of a single molecule. On the other hand, the binding energy is a property that is related to the ligand-receptor interaction that engages a population (ensemble) of molecules. It is worth mentioning here that recent advances in technology have made the so-called single-molecule biology system more and more popular $(18,19)$, which extends this problem to also include the property (single molecule vs. molar) representations. Accordingly, the uncertainty of LE comes from the ill-defined chemistry and not mathematics, because fragments (1 HAC or 1 Dalton) do not have a real molar representation. Interestingly, however, a mole of Daltons virtually represents $1 \mathrm{~g}(13,14)$.

A high LE preference for small ligands $(13,14)$ fits into the recent trends in pharma that favor small molecular ligands (the so-called slim pharma concept) (20), which have advantageous drug-likeness profiles (21). Therefore, LE performs unexpectedly well if used as guideline filters during the hit and lead optimization (22-25) despite the uncertainty in its physical meaning $(13,14)$. This uncertainty causes effects that have been interpreted as being unexpected and paradoxical. Basically, the LE trend could not be understood. To illustrate the problems, the development of the empirical formula of the socalled size-independent LE (SILE) can be cited here (26). The analogy of LE to car fuel efficiency can even better illustrate a confusion (12).

In this publication, we show that a more complete understanding of the LE enables rational predictors for molecular design to be defined. In practice, a low HAC and high potency indicate attractive drug candidates. This suggests an interaction between the HAC and potency which in the most general meaning will be represented here by $\mathrm{pAC}_{50}$. Statistically, LE probes the reciprocal interaction between $\mathrm{pAC}_{50}$ (negative logarithm of the active concentration) and 1/HAC. In turn, we confronted this with a direct multiplicative HAC and potency association. In particular, we analyzed the physical meaning of such a product ligand efficiency (PLE) and tested the behavior of the PLE on data from the PubChem and ChEMBL potency databases as well as on the selected series of drug and drug candidates $(1,25)$. Finally, the most important function of LE is its use as a guideline during the hit and lead optimization (22-25). In such functionality, the association of $\mathrm{AC}_{50}$ and $\mathrm{HAC}$ is just a scoring function enabling decision making in the drug pipeline. Accordingly, we defined a novel flexible predictor SCORE that is capable of adaptably scoring the development potential of drug candidates that is related to potency and the HAC.

\section{EXPERIMENTAL}

\section{Ligand binding: a potency vs. single biology, ligand} efficiency (LE) and binding efficiency index (BEI)

Measuring the binding effects of ligands is a complex problem. Accordingly, a ligand molecule interacts with a receptor to produce a signal. Actually, recent technological development has provided us with the possibility to directly measure these systems using the so-called single biology approaches $(18,19)$. However, historically, this representation has not been available for direct observations. Therefore, the so-called potency is commonly used to describe ligand-receptor systems. A collection of molecules interacts with a collection of receptors. In this model, a signal is produced by the competitive mechanism of ligand and receptor involvement thereby producing a well-known sigmoid-like potency signal, which is usually denoted by the so-called inhibitory concentration $\mathrm{IC}_{50}$. Ligand efficiency, LE, is another metric that has recently been proposed as an intensive representation of ligand binding, which originated in an effort to identify the maximal ligand affinities (5).

LE or BEI are calculated by a simple calculation of the proportion of binding properties to the molecular size that is denoted by the non-hydrogen atoms (the so-called heavy atom counts: HAC) or molecular weight (MW). In practice, LE has been of special interest in drug design. The astonishing successes of LE $(1,12)$ were confronted with a series of skeptical analyses that argue that the mathematics of LE is incorrect (9-11). The behavior of LE function has been widely investigated. For example, the nature of binding sites and the target class is likely to have an impact on ligand efficiency (for instance, inhibitors of protein-protein interactions versus enzyme inhibitors). Similarly, different mechanisms of activity should be treated separately for ligand efficiency purposes, because covalent inhibitors can 
have very high ligand efficiency values. In addition, larger, more optimized molecules may have smaller ligand efficiencies, which is due in part to the fact that such compounds have been optimized for properties other than binding to the target (for instance, pharmacokinetics, solubility, selectivity or cell permeability). It is vital to remember that ligand efficiency has limited value in late-stage lead optimization and that it should primarily be used in hit identification and hit-to-lead settings. Another factor that may have an important role could be assay limitations: many assays have a detection limit of $\mathrm{EC}_{50}$ of $\sim 1 \mathrm{~nm}$ due to signal-to-noise issues. Good ligand efficiency for large molecules would require subnanomolar potencies, which may not be recorded (or recorded correctly) because of assay limitations.

\section{Data}

We used the largest available potency data in the form of $\mathrm{AC}_{50}$ as is defined by the PubChem classification. Accordingly, we called all of the data that was used the active concentration, $\mathrm{AC}_{50}$. For PubChem, $\mathrm{AC}_{50}$ stands for the inhibitory concentration, $\mathrm{IC}_{50}$, the effective concentration, $\mathrm{EC}_{50}$, the cytotoxic concentration, $\mathrm{CC}_{50}$, the equilibrium dissociation constant, $\mathrm{K}_{\mathrm{i}}$, for the ligand that is determined directly in a binding assay using a labeled ligand or dissociation constant, $\mathrm{K}_{\mathrm{d}}$, for the ligand determined in inhibition studies. For ChEMBL the $\mathrm{AC}_{50}$ values are either the $\mathrm{IC}_{50}, \mathrm{~K}_{\mathrm{i}}$ or $\mathrm{K}_{\mathrm{d}}$ values. Binding energy and potency are related as described in (1).

In the context of the used data, we should remember that originally LE has been defined as the contribution of a non-hydrogen atom to the binding free energy. Thermodynamically, $K_{i}$ and $K_{d}$ values are therefore adequate measures for calculating $\mathrm{LE}$. $\mathrm{IC}_{50}$ and more importantly $\mathrm{EC}_{50}$ values are determined in functional measurements and in many cases, they do not change proportionally with the binding affinity. However, in practice usually $\mathrm{IC}_{50}$, $\mathrm{EC}_{50}$ and $\mathrm{K}_{\mathrm{i}}$ data are used as interchangeable values. Such a practice is especially necessary if we would like to probe the big data type statistics.

Physical meaning of reciprocal (LE) and multiplicative ligand efficiency (PLE)

Formally, LE probes reciprocal interaction of $\mathrm{pAC}_{50}$ and HAC. Physical meaning of LE was described in $(13,14)$. We are exploring here a direct multiplicative interaction of $\mathrm{AC}_{50}$ and PLE as the product $\mathrm{AC}_{50}$. HAC. Below we clear a meaning of PLE for molar ligand representation.

Accordingly:

$$
\mathrm{PLE}=\mathrm{AC}_{50} \cdot \mathrm{HAC}
$$

As MW $(\mathrm{kg} / \mathrm{mole}) / \mathrm{MW}(\mathrm{Da})=1$, PLE can be defined by:

$$
\begin{gathered}
\mathrm{PLE}=\mathrm{AC}_{50} \cdot \mathrm{HAC} \cdot(\mathrm{MW}(\mathrm{kg} / \mathrm{mole}) / \\
\mathrm{MW}(\mathrm{Da}))
\end{gathered}
$$

Because $\mathrm{AC}_{50}$ is a concentration-based metric that has the dimension of $(\mathrm{mol} / \mathrm{L})$, from eq. $1 \mathrm{a}$, we obtain the PLE dimension (unit), which is $(\mathrm{kg} / \mathrm{L})$. HAC/MW(Da). This means that the physical meaning of the PLE is the minimum inhibitory concentration (MIC) scaled to the HAC. Since the $\mathrm{AC}_{50}$ usually relates to the $50 \%$ inhibitory concentration multiplicative LE also relates to $\mathrm{MIC}_{50}$, which is given in $\mathrm{kg} / \mathrm{L}$.

\section{Size independent LE (SILE)}

SILE was calculated from equation: SILE $=\mathrm{AC}_{50} / \mathrm{HAC}^{0.3}$

\section{The SCORE estimator}

SCORE is defined as:

$$
\mathrm{SCORE}=\mathrm{a} \cdot \mathrm{pAC}_{50}+\mathrm{b} \cdot \mathrm{pHAC}
$$

if $\mathrm{a}$ and $\mathrm{b}=1$, then SCORE $=$ pPLE.

\section{Data acquisition and calculations}

All of the records along with their numerical values used in the analyses were downloaded from the ChEMBL and PubChem databases. Records repeating individual compounds were treated as independent entries. From PubChem, 2,435,467 records (download: August 2017, pubchem.ncbi. nlm.nih.gov) and from ChEMBL, 779,714 records (ChEMBL version 24, www.ebi.ac.uk) were downloaded from their Internet sites, respectively. The $\mathrm{pAC}_{50}$ data for drugs or drug fragments were taken from the literature as the $\mathrm{pIC}_{50}$ values $(1,25)$. The mean values for the drugs are the $\mathrm{HAC} 31 ; \mathrm{pIC}_{50}$ 8.12 and for the fragments, the HAC $15 ; \mathrm{pIC}_{50} 4.41$. A series of fragment to lead drug development projects reported in J. Med. Chem. were analyzed after the data presented in the references (27-29). Additionally, the data for the selected series of drugs collected in the Binding Database were analyzed. This includes: BindingBD, 570,927 records, PTaylaorLab, 180 records, USPatent, 210,254 records, 5HT, 830 records, or AChE, 726 records (download: December 2018, www.bindingdb.org) and Psychoactive Drug Screening Program PDSP database 22,273 records (download: December 2018, pdsp.unc.edu/databases).

\section{Binning}

To plot the pPLE and $\mathrm{pAC}_{50}$ vs. the HAC for ChEMBL and PubChem data, we used a binning method. In binning, data that fall into a given inter- 
val, a bin, are replaced by a value that is representative of that interval. Individual single HAC numbers define the size of the intervals, while the $\mathrm{AC}_{50}$ values are represented by their median value. For a HAC $>60$, where not enough data were available, the HAC was binned for each 15 HACs.

\section{RESULTS AND DISCUSSION}

The physical meaning of LE determines its preference for small ligands. The question is whether we can design a predictor that would balance more evenly the interaction between potency and the HAC. In practice, a low HAC and low $\mathrm{AC}_{50}$ (high potency) values indicate attractive drug candidates. Therefore, multiplicative interaction of potency and the HAC (Product LE: PLE) should be an informative estimator of the quality of drug candidates. A simultaneous decrease in the HAC and $\mathrm{AC}_{50}$ will decrease the PLE, and vice versa, their increase will increase the PLE. Accordingly, both terms in the PLE act cooperatively. Interestingly, besides their relation to a single molecule, ligand efficiency estimators are evidently associated with substances and their properties (Compare Materials and Methods). Firstly, this proves the design concept for the PLE, which was shown to be related to the minimum inhibitory concentration (MIC) because the MIC is an obvious property measure that is related to the activity of a compound. Secondly, this drew our attention to the fact that in designing a metric that can be used to evaluate drug candidates, we should observe both its role as a synthetic descriptor that optimizes the balance between its activity and the HAC and its potential physical meaning, which originates from the descriptor-property interplay.
An interesting problem will be to analyze LE changes in the drug development pipeline. Johnson et al., observed that for the data published in J. Med. Chem. that "LE decreased during optimization for only a minority of examples." He indicated however, that "this finding should be treated with caution because the data analyzed here are biased toward publishable F2L campaigns." (27). To conclude LE in the F2L can both increase or decrease. However, can we indicate the key factors limiting this effect? A question is if we should expect any regularity for a relatively small population of ligands described in the reference (27). The similarity paradox claims that even the smallest structural change can result in the substantial activity changes; therefore; the regularity will rather be surprising. In Figure 1 we reanalyzed the F2L data (27-29) plotting the change of $\mathrm{AC}_{50}$ related values of $\Delta \mathrm{pAC} \mathrm{C}_{50}, \Delta \mathrm{LE}$ or $\triangle \mathrm{pPLE}$ in the course of F2L conversion as a function of HAC of the original fragments. As expected there is no correlation between the $\triangle \mathrm{pAC}_{50}$, and $\triangle \mathrm{pPLE}$ and $\mathrm{HAC}$ for fragments. In turn, $\triangle \mathrm{LE}$ obviously indicate an increasing trend vs. HAC for fragments. The lower the HAC of the fragment is the lower also is the gain in LE for the resulted lead. For a low HAC fragment any $\mathrm{AC}_{50}$ gain by the lead cannot balance the HAC contributing into the LE value of the fragment by means of the hyperbolic $1 / \mathrm{HAC}$ term. This clearly illustrates the dominating influence of HAC into LE, which can be explained by non-Avogadro LE statistics $(13,14)$. A small population of the F2L could not necessarily be generally representative; therefore; we focus below on the large potency databases to explore scoring potential of efficiency functions. A common mathematical representation for $\mathrm{AC}_{50}$ is its negative $\log$ scale, $\mathrm{pAC}_{50}$, in which higher values of $\mathrm{pAC}_{50}$ indicate an exponentially greater potency.
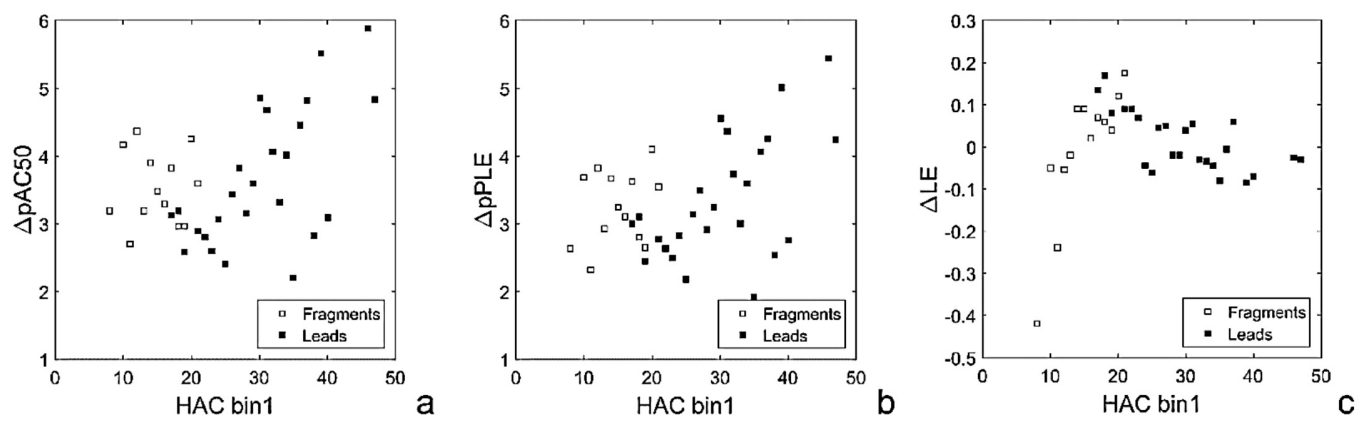

Figure 1 . The dependence of the difference between the lead and fragment potency measured as $\Delta \mathrm{pAC} \mathrm{C}_{50}$ (a) $\Delta \mathrm{pPLE}$ (b) or $\Delta \mathrm{LE}$ (c) as a function of HAC during F2L development reported in Journal of Medicinal Chemistry 2015-2017, indicated both for fragments and leads. Data after (27-29) 

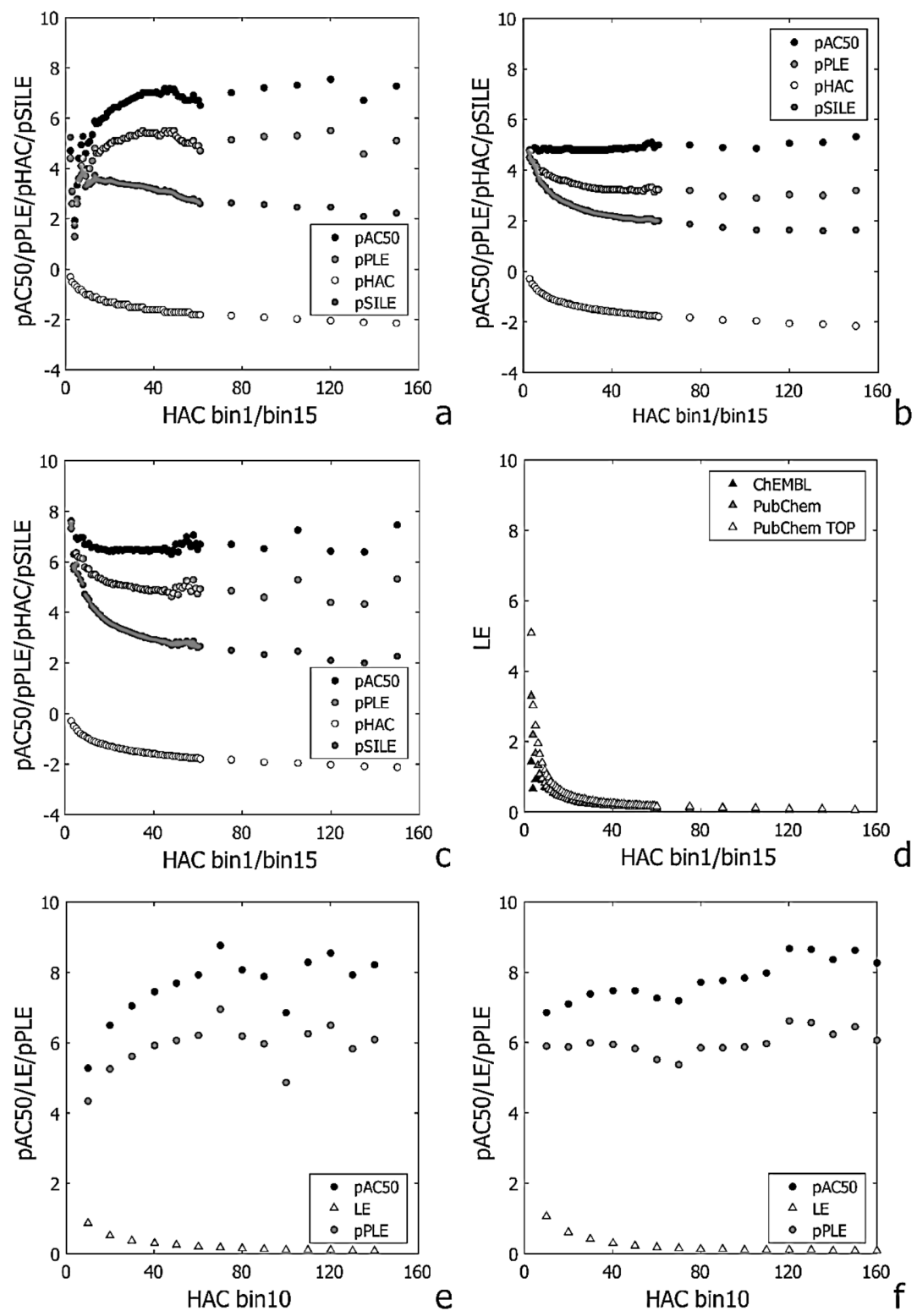

Figure 2. The dependence of the median pPLE on HAC for data from ChEMBL (a), PubChem (b), and a PubChem subpopulation with $\mathrm{pAC}_{50}>6$ (c). pPLE can be defragmented to median $\mathrm{pAC}_{50}$ (black dots) and pHAC (-logHAC, white dots) which is additionally illustrated in (a-c). For comparison we show pSILE (size-independent LE) in (a-c), the median LE for all data of ChEMBL, PubChem or a PubChem subpopulation of $\mathrm{pAC}_{50}>6(\mathrm{~d})$ and $\mathrm{AC}_{50}$ vs. HAC for a series of patented drug candidates (e) or psychoactive PDSP drugs (f)

Accordingly, we used the analogous pPLE scale (a higher pPLE indicates better quality) to analyze the PLE trends. Because the logarithm of a product is the sum of the logarithms, pPLE can be defragmented to its $\log \mathrm{HAC}(\mathrm{pHAC})$ and $\mathrm{pAC}_{50}$ components as is shown in Figures 2a-c. In Figure 2a, the ChEMBL data show an increase in $\mathrm{pAC}_{50}$ until a value of approximately 50 for the HAC. At the same time, the interaction between $\mathrm{pAC}_{50}$ and $\mathrm{pHAC}$ is clearly revealed in the pPLE which optimum is shifted slightly towards the lower HAC values in comparison to the maximum of $\mathrm{pAC}_{50}$ plot. In particular, for the pPLE plot the maximum at ca. 30-50 HAC is broader while the depression for high HAC (160) is higher. In Figures $2 \mathrm{~b}$ and $2 \mathrm{c}$, we illustrate the PubChem data, which shows that $\mathrm{pAC}_{50}$ is a constant function of the HAC (Fig. 2b), thus indicating that potency is not generally a function of the molec- 
ular size, if the probed population of the active compounds is large enough. If so, lower HAC, less complex ligands are better drug candidates, then pPLE properly indicates the lower HAC as statistically better. In turn, for the most active PubChem data (ligands of $\mathrm{pAC}_{50}>6$ ), $\mathrm{pAC}_{50}$ decreased with the HAC (Fig. 2c), thus indicating that the probability of the proper ligand-target fit decreases with an increasing molecular size for this high activity ligands. In both cases (Figs. 2b-c), the lowest HAC indicated the optimum candidates, which was correctly predicted by the pPLE. Accordingly, all possible interaction scenarios of $\mathrm{pAC}_{50}$ vs. HAC (Figs. $2 \mathrm{a}-\mathrm{c})$ were correctly predicted by the pPLE function. In turn, the LE vs. the HAC relationship for all of the data plotted together was very similar, always indicating the lowest HAC as optimal (Fig. 2d). In other words, as a predictor LE emphasizes a low HAC more than a high degree of potency. A series of $\mathrm{pAC}_{50}$ vs. HAC relationships were explored and plotted in Figures 2e-f. Accordingly, we show a series of US patented drug candidates (Fig. 2e). A typical increase trend of $\mathrm{AC}_{50}$ and pPLE vs. HAC can be observed for HAC below 50. In turn for psychoactive PDSP drugs the increase of $\mathrm{pAC}_{50}$ vs. HAC was not enough strong to result in the increasing trend of pPLE (Fig. 2f). For a comparison in Figures 2 a-c we illustrated a plot of SILE which is somewhere between pPLE and pHAC.

In Figure 3a, we show the application of the pPLE to evaluate a series of drugs (1) and fragmentlike drug candidates (25). Regardless of the HAC range, the pPLE value was always higher for the drugs than for the fragments. There was a clear separation of the cluster of drugs from the cluster of fragments. Furthermore, all of the drugs had a higher PLE value than the fragments. Accordingly, the
pPLE could be a well-balanced predictor that can clearly indicate the development from fragments to drugs. In turn, Figure $4 \mathrm{~b}$ illustrates the LE statistics for the same data. An analysis of the plot of LE vs. HAC shows that for a given HAC above 20, the drugs had a slightly higher value of LE than the fragments. However, for HAC values below 20, LE for the fragments is higher than any drug can achieve.

The most important function of LE is its use as a guideline during the hit and lead optimization (2225) where it forces slim pharma at the same time preventing molecular obesity. The mechanism for that was shown in Figure 1. In such a functionality the association of $\mathrm{AC}_{50}$ and $\mathrm{HAC}$ is just a scoring function enabling decision making in the drug pipeline. Below we designed a versatile function can be formed in which the effect of the pHAC and $\mathrm{pAC}_{50}$ components can be tuned by additional a and b parameters:

$$
\mathrm{SCORE}=\mathrm{a} \cdot \mathrm{pAC}_{50}+\mathrm{b} \cdot \mathrm{pHAC}
$$

if $\mathrm{a}$ and $\mathrm{b}=1$, then SCORE $=$ pPLE.

Mathematically, the numerical values of HAC are usually between 1 and 200 , while $\mathrm{AC}_{50}$, usually is in the range of 1 to 7 ; thus, $1 / \mathrm{HAC}$ clearly dominates in LE. In other words, LE as a predictor emphasizes low HAC more than high potency. In turn, $\mathrm{AC}_{50}$ (the range of $10^{1}$ to $10^{7}$ ) dominates in pPLE. SCORE was designed as a predictor for the more balanced scoring of the interaction between $\mathrm{AC}_{50}$ and the HAC. This function can be adjusted flexibly to change the relative SCORE rank of the fragments and drugs themselves as well as fragments and drug clusters. In particular, changing the $a$ and $b$ parameters can model different fragments to drug development strategies. Accordingly, depending on the preferences we can fine tune the SCORE
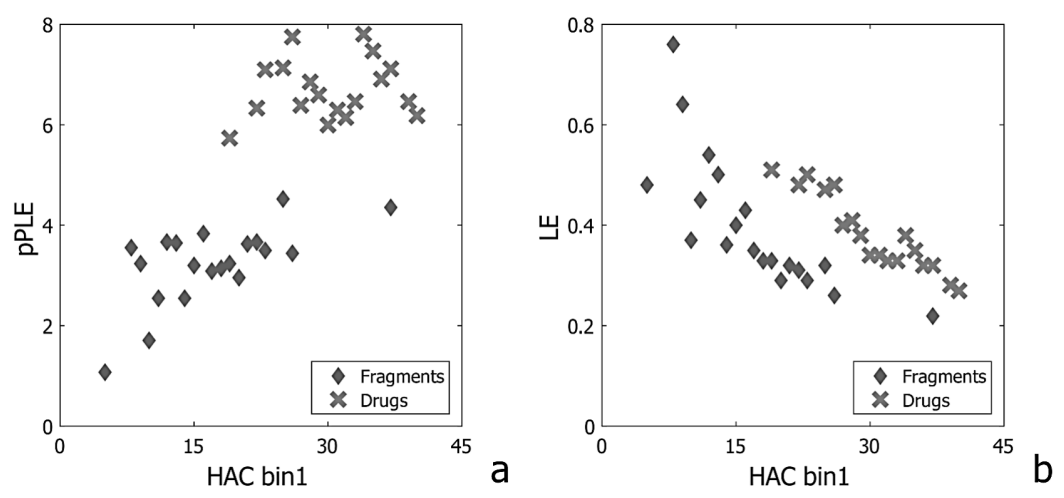

Figure 3. The dependence of pPLE (a), LE (b) on HAC for drug fragments and drugs 

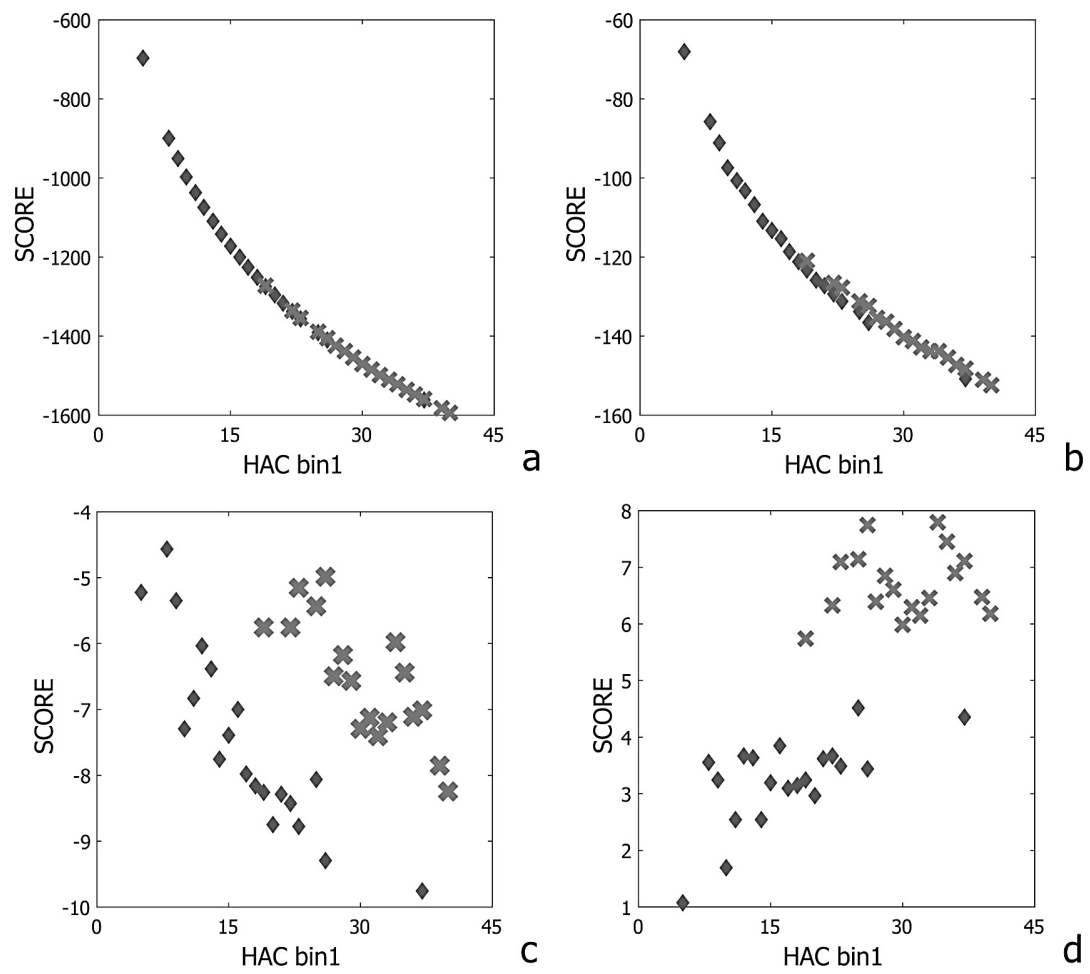

Figure 4. The dependence of SCORE on HAC for drug fragments and drugs for the different $a$ and $b$ values: $\mathrm{a}=1, \mathrm{~b}=1000$ (a); a $=1, \mathrm{~b}$ $=100(\mathrm{~b}) ; \mathrm{a}=1, \mathrm{~b}=10(\mathrm{c}) ; \mathrm{a}=1 \mathrm{~b}=1(\mathrm{~d})$

function in order to adopt the strategy that is required by a specific drug development project.

\section{CONCLUSION}

We explained here the molecular reason for why LE prefers a low HAC to a level that practically masks the influence of $\mathrm{AC}_{50}$. As drug design is a complex problem and drug-likeness prefers small molecules similar to LE, therefore, LE performs unexpectedly well in drug development. An important advantage of LE is that if used as a molecular filter for decision making it prefers small molecules and currently small molecules are among the most promising targets of slim pharma due to the advantageous drug-like profiles. It is however, not only the above mentioned feature of LE that contributed to the efficiency of LE. We have a number of methods for the estimation (prediction) of drug-likeness, e.g., ADMET or Lipinski's rule of five. Although usually these methods are described as drug-like property filters expected to provide a higher success ratio at the advanced development stages based on the values of the molecular feature that are typical for drugs, precisely speaking, these filters cannot usually be based on the properties that are measured because molecules that are being designed at this stage of projects are not available for experimental measurements. Accordingly, they are molecular descriptors that are calculated for the molecular representations in order to predict the desired properties of the respective substances. The most obvious example is $\log \mathrm{P}$, in which based on ca. $30000 \log \mathrm{P}$ measurements, a regression model is built to provide predictions for any real or virtual molecule. In comparison, LE is a property related function, because we should know a real biological activity to calculate LE. Accordingly, we are not assuming or predicting positive activity, but we do know this fact. This is what makes LE the most reliable marker of drug-likeness.

We showed here that the product ligand efficiency (PLE), which is the product of the $\mathrm{pAC}_{50}$ values with the HAC could be an informative drug candidate estimator in which $\mathrm{pAC}_{50}$ and the HAC were balanced more evenly. At the same time, the physical meaning of the PLE and other efficiency estimators drew our attention to the relation between vari- 
ous chemical representation of chemical compounds, i.e., molar properties, vs. descriptors vs. single molecule properties. On the other hand, finding ligands in drug design can be illustrated by playing between their matching vs. binding ability. In this context, the multiplicative PLE statistics indicate that playing between matching and binding is especially promising at around 30-50 HAC, where PLE takes the maximum value. Moreover, the SCORE predictor was designed for the flexible fine tuning of the ligand $\mathrm{HAC}$ vs. the $\mathrm{IC}_{50}$ interaction as may be required by a specific drug development project.

\section{Acknowledgments}

Financial support was received from NCBR (Warsaw): ORGANOMET No: PBS2/A5/40/2014, TANGO1/266384/NCBR/2015. The anonymous Reviewer for the indication of the LE function behavior.

\section{Conflicts of interest}

The authors declare no competing financial interest.

\section{REFERENCES}

1. Hopkins A.L., Keseru G.M., Leeson P.D., Rees D.C., Reynolds C.H.: Nat. Rev. Drug Discov. 13, 105 (2014).

2. Williams G., Ferenczy G.G., Ulander J., Keseru G.M.: Drug Discov. Today. 22, 681 (2017).

3. Hann M.M., Leach A.R., Harper G.: J. Chem. Inf. Comput. Sci. 41, 856 (2001).

4. Zartler E.R., Shapiro M.J.: Curr. Opin. Chem. Biol. 9, 366 (2005).

5. Kuntz I.D., Chen K., Sharp K.A., Kollman P.A.: Proc. Natl. Acad. Sci. U.S.A. 96, 9997 (1999).

6. Reynolds C.H., Bembenek S.D., Tounge B.A.: Bioorganic Med. Chem. Lett. 17, 4258 (2007).

7. Reynolds C.H., Tounge B. A., Bembenek S. D.: J. Med. Chem. 51, 2432 (2008).

8. Reynolds C.H., Reynolds R.C.: J. Chem. Inf. Model. 57, 3086 (2017).

9. Shultz M.D.: ACS Med. Chem. Lett. 5, 2 (2014).
10. Shultz M.D.: Bioorganic Med. Chem. Lett. 23, 5980 (2013).

11. Zhou H., Gilson M.: Chem. Rev. 109, 4092 (2009).

12. Murray C.W., Erlanson D.A., Hopkins A.L., Keseru G.M., Leeson P.D. et al.: ACS Med. Chem. Lett. 5, 616 (2014).

13. Polański J., Tkocz A., Kucia U.: J. Cheminform. 9, 49 (2017).

14. Polański J., Tkocz A.: J. Chem. Inf. Model. 57, 1321 (2017).

15. Sheridan P.R.: J. Chem. Inf. Model. 56, 2253 (2016).

16. Scott J., Waring M.: Bioorganic Med. Chem. 26, 3006 (2018).

17. Polański J.: in Silico Encyclopedia of Bioinformatics and Computational Biology, Ranganathan S., Gribskov M., Nakai H., Schonbach Ch., Eds, Vol. 2, pp. 601-618, Elsevier 2019.

18. Knight A.: Single Molecule Biology. 1st ed., Academic Press, New York 2009.

19. Leake M.: Philos. Trans. R Soc. Lond. B Biol. Sci. 368, 1611 (2013).

20. Hann M.: MedChemComm 2, 349 (2011).

21. Shultz M.D.: J. Med. Chem. 62, 1701 (2019).

22. Mignani S., Rodriguez J., Tomas H., Jalal R., Parvinder P.S. et al.: Drug Discov. Today 23, 605 (2018).

23. Meanwell N.A.: Chem. Res. Toxicol. 29, 564 (2016).

24. Cavalluzzi M.M., Mangiatordi G.F., Nicolotti O., Lentini G.: Expert Opin. Drug Discov. 12, 1087 (2007).

25. Schultes S., de Graaf C., Haaksma E., de Esch I., Leurs R. et al.: Drug Discov. Today Technol. 7, e157 (2010).

26. Nissink J.: J. Chem. Inf. Model. 49, 1617 (2009).

27. Mortenson P.N., Erlanson D.A., de Esch I.J.P., Jahnke W., Johnson C.N.: J. Med. Chem. 61, 1774 (2018).

28. Johnson C.N., Erlanson D.A., Murray C.W., Rees D.C.: J. Med. Chem. 60, 89 (2016).

29. Johnson C.N., Erlanson D.A., Jahnke W., Mortenson P.N., Rees D.C.: J. Med. Chem. 61, 1774 (2018).

Received: 6.01 .2019 